\title{
Design of Fuzzy Self - tuning PID Controller for Master-Slave Manipulator Force Sensing System
}

\author{
Xiao-Dong LIANG ${ }^{1, a}$, Zhong-Kai Chen ${ }^{1, b}$, Ye Sun ${ }^{1, c}$, Hong-Bo LIAO $^{1, d}$ \\ ${ }^{1}$ Northwest Institute of Nuclear Technology, xi'an 710024,China \\ axiaodoudong@126.com, ${ }^{b}$ chzhk@mail.ustc.edu.cn, ${ }^{c}$ 123sunye@163.com, \\ 13975890826@163.com
}

\begin{abstract}
Keywords: master-slave manipulator force sensing system; fuzzy self-tuning PID controller; matlab/simulink

Abstract. For the problems about large disturbances, delays and nonlinear of master-slave manipulator force sensing system, the paper designed a fuzzy self-tuning PID controller and carried out simulation analysis by matlab/simulink. The result showed that the designed controller can effectively improve the dynamic and steady state of the force control system.
\end{abstract}

\section{Introduction}

In the industrial process, PID control is widely used because of its simple principle, convenient operation and strong robustness. But as for some systems with large disturbances, delays and nonlinear, it is difficult to establish the precise mathematical model by traditional PID controller. Parameter tuning is difficult and the best parameters are easy to drift, so the controller parameters must be adjusted off-line or on-line to obtain reasonable values. Fuzzy self-tuning PID control combines the traditional PID control and fuzzy control and the advantages of both sides. It can adjust the parameters of PID online and in real time, which is a kind of advanced control system at present $^{[2,3,4]}$.

Aiming at the problems about large disturbances, delays and nonlinear of master-slave manipulator force sensing system, we designed a fuzzy self-tuning PID controller and carded out simulation analysis by matlab/simulink. The result showed that the designed controller can effectively improve the dynamic and steady state of the force control system.

\section{Introduction of the master-slave manipulator force sensing system}

The master-slave manipulator consists of three parts: the master hand, the slave hand and the control system, as shown in figure 1. The operator passes the joint movement command through the master hand to control the movement of the slave hand. When the slave hand reaches the working position for clamping operation, the clamping handle of the master hand output signal to control the slave hand gripper closed, thus the clamping and handling operation is done. The control system is mainly responsible for collecting position information and force information, driving the power components, coordinating and controlling the master and slave manipulator.
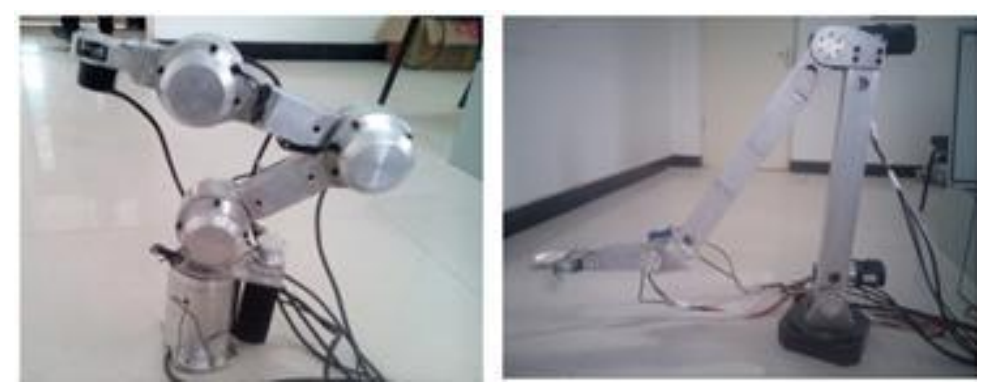

Fig. 1 Physical Drawing of The Master-Slave Manipulator 
Master-slave manipulator force control system can sense the clamping force change of the salve hand in real time and realize the feedback of the clamping force, so that the operator has a sense of clamping force. It is mainly composed of force sensor, motion controller, computer, master hand force feedback handle, wire rope, motor controller and motor. The system control block diagram is shown in figure 2.

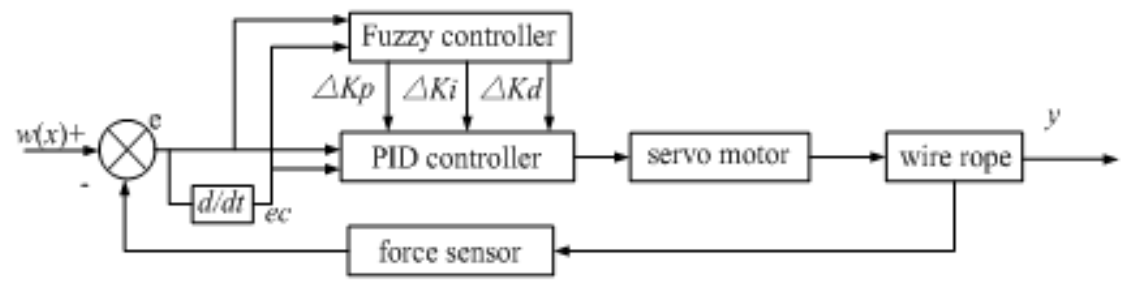

Fig. 2 Block Diagram of Force Control System

It can be seen from figure 2, in the force control system the control quantity is the tension at the end of the wire rope, and the force servo control is achieved through that the AC servo motor drives rope wheel to pull the wire rope. The tension of the wire rope is the key quantity and terminal quantity of the control, and the pulling force is detected by the tension and pressure sensor.

\section{Controller Design}

Fuzzy control is a kind of control method using fuzzy set theory. The rationality and advantages of fuzzy control are as follows: Fuzzy control proposes a new mechanism of control law based on rule language, and provides a new theory and method for system and device with uncertain model parameters, time variability and non-linearity.

Determination of membership function about input and output quantity. According to the PID parameter self-tuning principle, the fuzzy self-tuning PID controller is designed as a double-input three-output type, the input quantity is the force control signal deviation $e$ and the deviation change rate $e c$, where the deviation $e$ is equal to the difference between the two measured wire rope tension signals, and the deviation change rate $e c=e k$-ek-1, the output is $\Delta K_{p}, \Delta K_{i}, \Delta K_{d}$.

Force deviation $e:[-6,6]$; deviation change rate $e c$ : [- 6,6]; output $\Delta K_{p}:[-0.6,0.6]$

Output $\Delta K_{i}:[-6,6]$; output $\Delta K_{d}:[-0.06,0.06]$

The fuzzy subset in the fuzzy controller is expressed by \{negative big, negative middle, negative small, zero, positive small, positive middle, positive big\}:

$E=\{\mathrm{NB}, \mathrm{NM}, \mathrm{NS}, \mathrm{Z} 0$, PS, PM, PB $\}$

$E C=\{\mathrm{NB}, \mathrm{NM}, \mathrm{NS}, \mathrm{Z} 0, \mathrm{PS}, \mathrm{PM}, \mathrm{PB}\}$

$\Delta K_{p}, \Delta K_{i}, \Delta K_{d}=\{\mathrm{NB}, \mathrm{NM}, \mathrm{NS}, \mathrm{Z} 0, \mathrm{PS}, \mathrm{PM}, \mathrm{PB}\}$ 

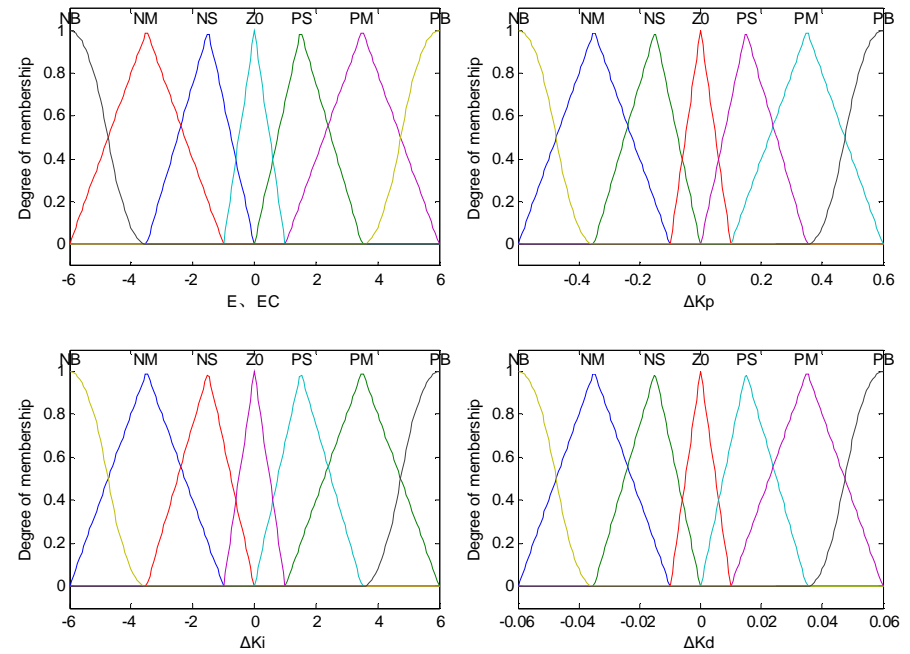

Fig. 3 Membership Function Curve of $E, E C, \Delta K_{p}, \Delta K_{d}, \Delta K_{i}$

Determine the fuzzy control rules. The control rules are in the form of if $e$ and $e c$ then $\Delta K$. According to expert rules and computer simulation debugging, the final determined fuzzy control rules of $\Delta \mathrm{Kp}, \Delta \mathrm{Ki}$ and $\Delta \mathrm{Kd}$ are shown in table $1 \sim 3$.

Table 1 Fuzzy Control Rules Table of Scale Coefficient

\begin{tabular}{|c|l|l|l|l|l|l|l|l|}
\hline \multicolumn{2}{|c|}{$\Delta \boldsymbol{K}_{\boldsymbol{p}}$} & \multicolumn{7}{|c|}{$\boldsymbol{E}$} \\
\cline { 2 - 9 } & $\boldsymbol{N B}$ & $\boldsymbol{N M}$ & $\boldsymbol{N S}$ & $\boldsymbol{Z O}$ & $\boldsymbol{P S}$ & $\boldsymbol{P M}$ & $\boldsymbol{P B}$ \\
\hline \multirow{7}{*}{$\boldsymbol{E} \boldsymbol{C}$} & $\boldsymbol{N B}$ & PB & PB & PM & PM & PM & PM & PB \\
\cline { 2 - 9 } & $\boldsymbol{N M}$ & PB & PB & PM & PM & PM & PM & PB \\
\cline { 2 - 9 } & $\boldsymbol{N} \boldsymbol{S}$ & PM & PM & PM & Z0 & PM & PS & PM \\
\cline { 2 - 9 } & $\boldsymbol{Z O}$ & PM & PS & PS & PS & PM & PB & PB \\
\cline { 2 - 9 } & $\boldsymbol{P S}$ & PS & PS & Z0 & NS & NS & NM & NM \\
\cline { 2 - 9 } & $\boldsymbol{P M}$ & Z0 & Z0 & NS & NM & NM & NM & NB \\
\cline { 2 - 10 } & $\boldsymbol{P B}$ & Z0 & NS & NS & NM & NM & NB & NB \\
\hline
\end{tabular}

Table 2 Fuzzy Control Rules Table of Integral Coefficient

\begin{tabular}{|c|c|c|c|c|c|c|c|c|}
\hline \multirow{2}{*}{\multicolumn{2}{|c|}{$\Delta \boldsymbol{K}_{i}$}} & \multicolumn{7}{|c|}{$E$} \\
\hline & & $N B$ & $N M$ & $N S$ & $Z 0$ & $P S$ & $P M$ & $P B$ \\
\hline \multirow{7}{*}{$\boldsymbol{E}$} & $N B$ & NB & NB & NB & NB & PM & PB & PB \\
\hline & $N M$ & NB & NB & NM & NB & $\mathrm{PM}$ & PB & PB \\
\hline & $N S$ & NM & NM & NM & NB & PS & PM & PM \\
\hline & $Z 0$ & NB & $\mathrm{NS}$ & $\mathrm{NM}$ & $\mathrm{NM}$ & PS & PS & PB \\
\hline & $P S$ & $\mathrm{NS}$ & $\mathrm{NS}$ & NM & NM & PS & PM & PM \\
\hline & $P M$ & $\mathrm{ZO}$ & $\mathrm{ZO}$ & PS & NM & PM & PB & PB \\
\hline & $P B$ & $\mathrm{ZO}$ & $\mathrm{ZO}$ & PS & $\mathrm{NM}$ & PB & PB & PB \\
\hline
\end{tabular}

Table 3 Fuzzy Control Rules Table of Differential Coefficient

\begin{tabular}{|c|c|l|l|l|l|l|l|l|}
\hline \multicolumn{2}{|c|}{$\Delta \boldsymbol{K}_{\boldsymbol{d}}$} & \multicolumn{7}{|c|}{$\boldsymbol{E}$} \\
\cline { 2 - 9 } & $\boldsymbol{N B}$ & $\boldsymbol{N M}$ & $\boldsymbol{N S}$ & $\boldsymbol{Z O}$ & $\boldsymbol{P S}$ & $\boldsymbol{P M}$ & $\boldsymbol{P B}$ \\
\hline \multirow{4}{*}{$\boldsymbol{E} \boldsymbol{C}$} & $\boldsymbol{N B}$ & PB & PM & PB & PB & PB & Z0 & NB \\
\cline { 2 - 9 } & $\boldsymbol{N M}$ & PM & PS & PM & PM & PM & NS & NB \\
\cline { 2 - 9 } & $\boldsymbol{N S}$ & PS & Z0 & PS & PS & PS & NM & NB \\
\cline { 2 - 9 } & $\boldsymbol{Z O}$ & NB & NM & NM & NS & Z0 & PS & PM \\
\cline { 2 - 9 } & $\boldsymbol{P S}$ & NB & NB & PS & PS & PS & Z0 & PS \\
\hline
\end{tabular}




\begin{tabular}{|l|l|l|l|l|l|l|l|l|}
\hline & $\boldsymbol{P M}$ & NB & NS & PM & PM & PM & PS & PM \\
\cline { 2 - 8 } & $\boldsymbol{P B}$ & NB & Z0 & PB & PB & PB & PM & PB \\
\hline
\end{tabular}

In the process of online self-tuning of PID parameters, the tension and pressure sensor can measure the actual tension pressure in real time, compare it with the set input value (expected value) to obtain the force deviation $e$, calculate $e k$-ek-1 and obtain the deviation rate $e c$. Set $e$ and $e c$ as the input of fuzzy controller, $K_{p}, K_{i}, K_{d}$ can be obtained through fuzzification, fuzzy rule selection and fuzzy clustering algorithm. According to the formula (1), we can obtain $K_{p}, K_{i}, K_{d}$, and the self-tuning PID control parameters, and then apply them to the control.

$$
\mu=\frac{\sum \mu\left(u_{i}\right) \cdot u_{i}}{\sum \mu\left(u_{i}\right)_{i}}
$$

Where: $u_{i}$ and $\mu\left(u_{i}\right)$ respectively represent the centroid and membership values of the symmetric membership functions.

Run Matlab, open the FIS editor, create * fis file, and select the control type as Mamdani type. "AND" operation adopts "prod", "OR" operation adopts "probor", fuzzy implication adopts "prod", fuzzy rule synthesis adopts "probor", defuzzification method adopts gravity method. The fuzzy controller and the membership function interface are shown in figure 4 and figure 5.

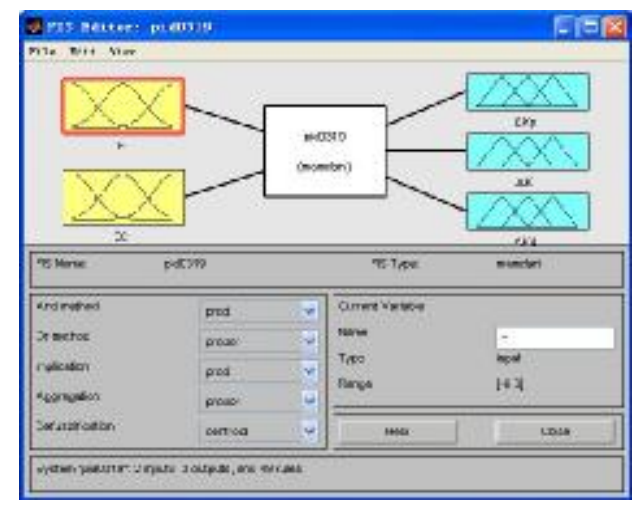

Fig. 4 Interface of Fuzzy Controller

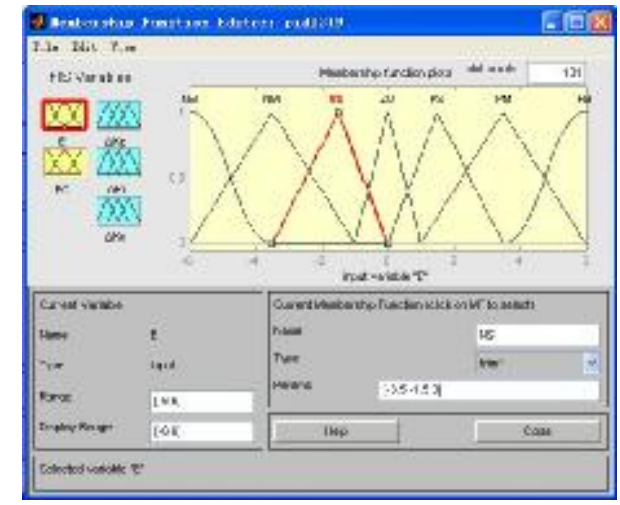

Fig. 5 Interface of Membership Function

Using the fuzzy rule editor, according to the control rules in table $1 \sim 3$ to edit, and get the rules of the input and output variables as shown in figure 6 , the curve surface of fuzzy relation is shown in figure 7 9.

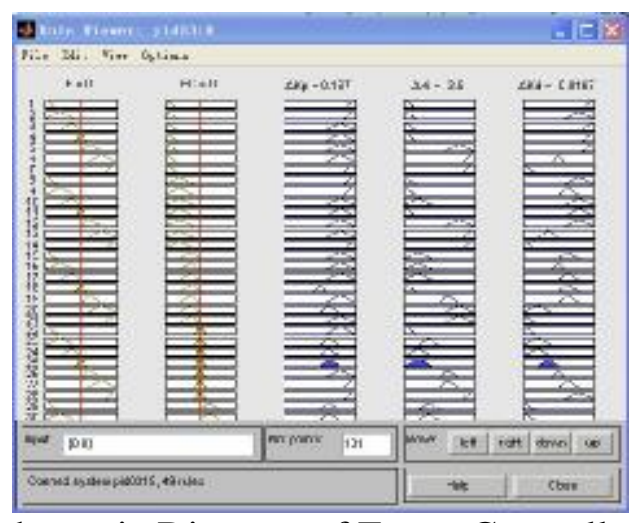

Fig. 6 Schematic Diagram of Fuzzy Controller Rule

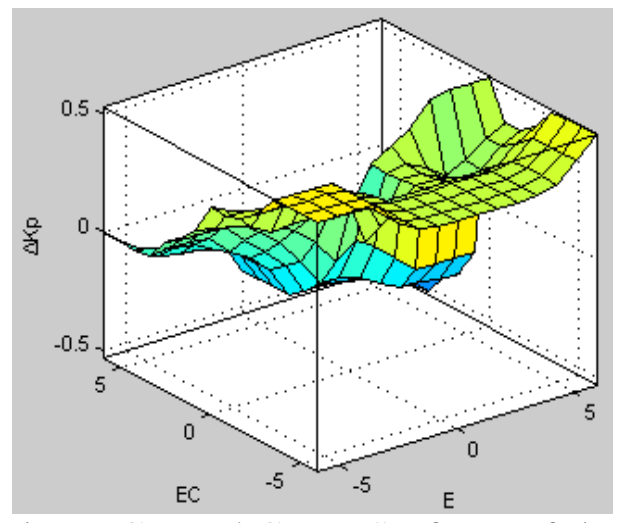

Fig. 7 Control Curve Surfaces of $\Delta K_{p}$ 


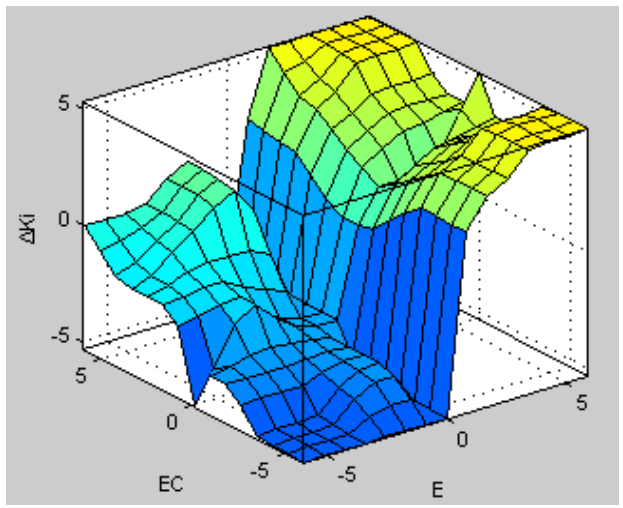

Fig. 8 Control Curve Surfaces of $\Delta \mathrm{Ki}$

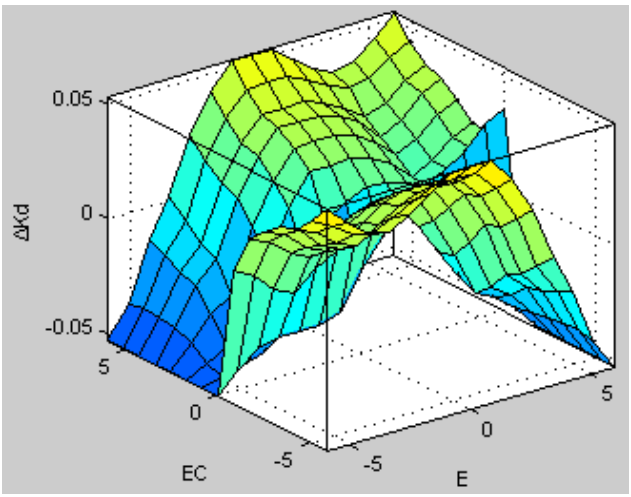

Fig. 9 Control Curve Surfaces of $\Delta K_{d}$

\section{Simulation analysis}

Construct the simulation model of the force system in the matlab / simulink, and drag the fuzzy logic controller module to the simulation model of the force system, and then carry out the simulation analysis, as shown in figure 10. Add the output $\Delta K_{p}, \Delta K_{i}, \Delta K_{d}$ of the fuzzy logic inference device which are weighted by the influence factor to the initial value of the PID parameter as the output of the PID controller. The parameters adjusted by the critical proportion method of the PID parameters are: $K_{p}=3.71, K_{i}=240, K_{d}=0.001$, the scale factors $\Delta p, \Delta i$ and $\Delta d$ are taken as 1 .

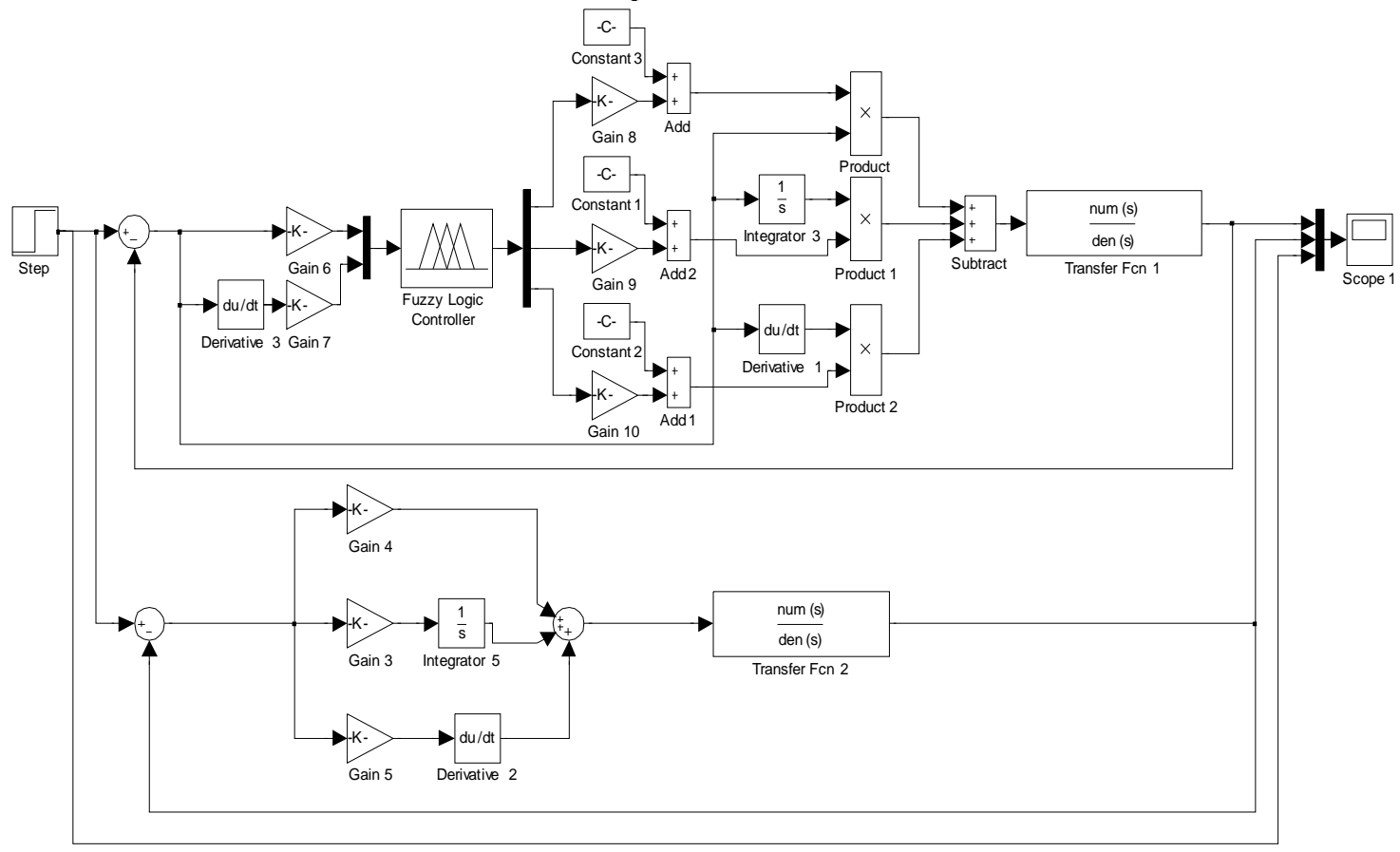

Fig. 10 Simulation Model of System Simulink

The adjusting curves of the self-tuning PID controller parameters $\Delta K_{p}, \Delta K_{i}, \Delta K_{d}$ are shown in figure 11-13. 


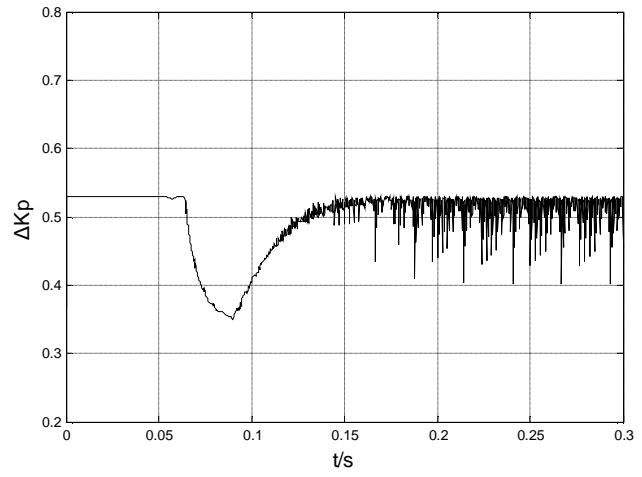

Fig.11 Adaptive Adjustment of $\Delta K_{p}$

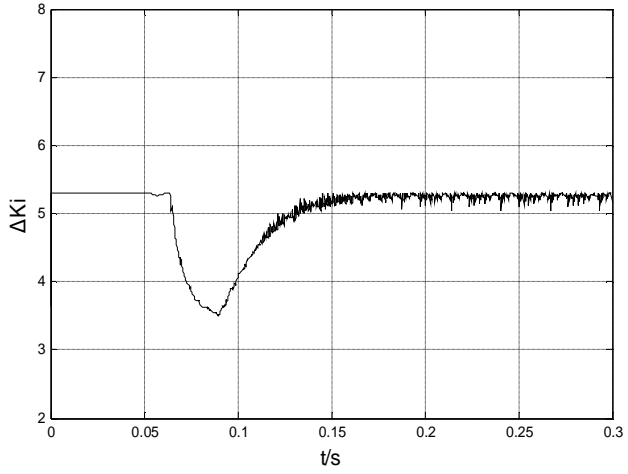

Fig.12 Adaptive Adjustment of $\Delta K$

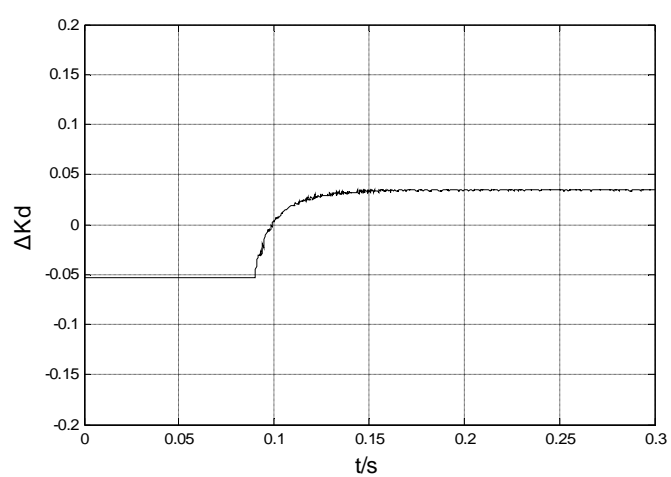

Fig.13 Adaptive Adjustment of $\Delta \mathrm{K}_{\mathrm{d}}$

Emulate the compared method between the output results of simple PID control and the output results of fuzzy self-tuning PID controller. The simulation results are shown in figure 14.

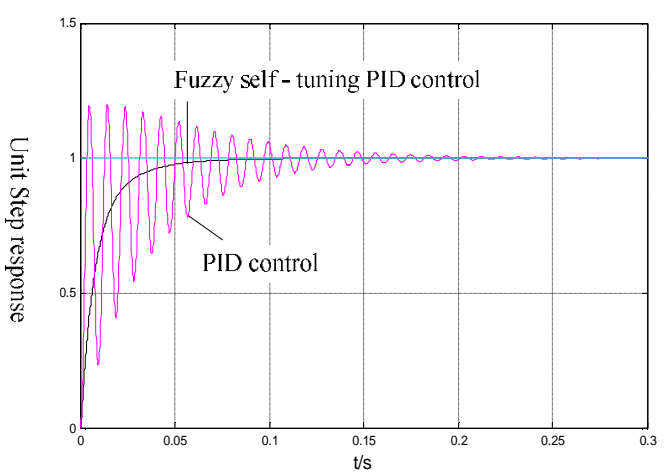

Fig. 14 Comparison of Unit Step Response

It can be seen from the figure 14 that compared with the traditional PID controller, the step response time of the system is shortened by about $50 \%$ by using the fuzzy self-tuning PID controller, with non-overshoot and small steady-state error.

\section{Result analysis}

Using the force sensing system of master-slave manipulator as the application background, and aiming at the existing problems about about large disturbances, delays and nonlinear in the system, we propose a design method of fuzzy self-tuning PID controller, and get the following conclusions through the simulation analysis.

(1) Compared with the traditional PID controller, the fuzzy self-tuning PID controller can shorten the step response time of the system by about $50 \%$, with non-overshoot and small steady-state error. 
(2) Compared with the traditional PID controller, the parameters fuzzy self-tuning PID controller has better robustness, and it can overcome the effect of large disturbance, nonlinear and other factors on the control accuracy, which makes the system maintain fast response speed and higher control accuracy.

\section{References}

[1] Chen Zhongkai. Research on master-slave manipulator clamping force servo system control [D]. University of Science and Technology of China, 2015.

[2] Huang Xiaoyu. Computer simulation of fuzzy self-tuning PID parameter controller based on MATLAB[J]. Automation and Instrumentation, 2001, 3:21-24.

[3] Gao Hongwei, Zhao Baoyong, Fu Xingwu.MATLAB simulation research of fuzzy self-tuning PID control strategy[J]. Electrical Drive Automation, 2002,24 (5): 21-24.

[4] Li Ping, Li Feng, Zhao Hu et al. Design and simulation of fuzzy self-tuning PID controller[J]. Journal of Instrumentation, 2004,25 (4): 264-267.

[5] Chen Jing, Pei Xiping, Shi Xiaoying, et al.Design and simulation of a fuzzy self-tuning PID controller[J]. Journal of Hubei Institute of Engineering, 2003, 18 (6): 30-32.

[6] Cheng Qijian. Research and application on fuzzy self-tuning PID controller[D]. Xi'an University of Technology, 2016.

[7] Wu Bin. Design and development of fuzzy self-tuning PID controller[D]. North China Electric Power University, 2015.

[8]Kwok D P,Tam P,Li C K,et al.Analysis and design of fuzzy PID control systems[C]. IEEE international Conference on Control, 1991, Vol. 2, pp: 955-960. 